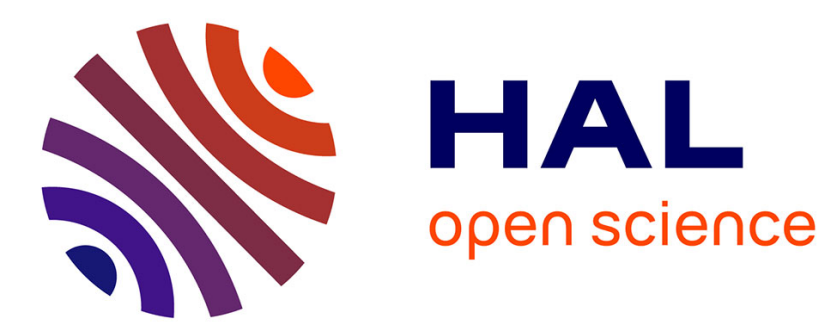

\title{
A space-time approach in digital image correlation: Movie-DIC
}

Gilles Besnard, Sandra Guérard, Stéphane Roux, François Hild

\section{To cite this version:}

Gilles Besnard, Sandra Guérard, Stéphane Roux, François Hild. A space-time approach in digital image correlation: Movie-DIC. Optics and Lasers in Engineering, 2011, 49, pp.71-81. 10.1016/j.optlaseng.2010.08.012 . hal-00521203

\section{HAL Id: hal-00521203 \\ https://hal.science/hal-00521203}

Submitted on 26 Sep 2010

HAL is a multi-disciplinary open access archive for the deposit and dissemination of scientific research documents, whether they are published or not. The documents may come from teaching and research institutions in France or abroad, or from public or private research centers.
L'archive ouverte pluridisciplinaire HAL, est destinée au dépôt et à la diffusion de documents scientifiques de niveau recherche, publiés ou non, émanant des établissements d'enseignement et de recherche français ou étrangers, des laboratoires publics ou privés. 


\title{
A space-time approach in digital image correlation: Movie-DIC
}

\author{
Gilles Besnard $^{\mathrm{a}, \mathrm{b}}$, Sandra Guérard ${ }^{\mathrm{b}, \mathrm{c}}$, Stéphane Roux ${ }^{\mathrm{b}, *}$, François Hild $^{\mathrm{b}}$ \\ ${ }^{a} C E A, D A M, D I F$, F-91297 Arpajon, France \\ ${ }^{b}$ LMT-Cachan, ENS Cachan / CNRS / UPMC / UniverSud Paris \\ 61 avenue du Président Wilson, F-94235 Cachan Cedex, France \\ ${ }^{c}$ now at Laboratoire de Biomécanique, Arts et Métiers ParisTech, ENSAM / CNRS, \\ 151 boulevard de l'Hôpital, F-75013 Paris, France
}

\begin{abstract}
A new method is proposed to estimate arbitrary velocity fields from a time series of images acquired by a single camera. This approach, here focused on a single spatial plus a time dimension, is specialized to the decomposition of the velocity field over rectangular shaped (finite-element) bilinear shape functions. It is therefore assumed that the velocity field is essentially aligned along one direction. The use of a time sequence over which the velocity is assumed to have a smooth temporal change allows one to use elements whose spatial extension is much smaller than in traditional digital image correlation based on successive image pairs. This method is first qualified by using synthetic numerical test cases, and then applied to a dynamic tensile test performed on a tantalum specimen. Improvements with respect to classical digital image correlation techniques are observed in terms of spatial resolution.
\end{abstract}

Keywords: Digital image correlation, displacement field, strain field, strain rate field, velocity field, video.

\footnotetext{
* Corresponding author

Email address: stephane.roux@lmt.ens-cachan.fr (Stéphane Roux)
} 


\section{Introduction}

In Solid Mechanics, digital imaging is used to detect and measure the motion and deformation of objects. From these observations follow various evaluation procedures of mechanical parameters [1]. To achieve this goal, different optical techniques are used [2]. Among them, Digital Image Correlation (DIC) is appealing thanks to its versatility in terms of scales ranging from nanoscopic $[3,4]$ to macroscopic $[5,6]$ observations with essentially the same type of algorithms.

DIC always involves a compromise between spatial resolution and uncertainty $[7,8]$. As the technique exploits the comparison of zones of interest, or elements between a deformed and a reference image, the information is carried by the pixels contained in those regions. A key characteristic is thus, $\beta$, the number of pixels per kinematic degree of freedom. On the one hand, low uncertainties call for a large $\beta$ (i.e., large elements), but the description of the displacement will be coarse, and hence maybe unsuited to capture rapidly varying displacement fields. The resulting systematic error may be prohibitive for a specific application. On the other hand, small elements may be more flexible to account for a complex displacement field, but as the information content, or $\beta$, is small, large uncertainties will result. This trade-off has to be solved for every application, depending on the "complexity" of the expected displacement field. However, one may have access to a large number of pictures thanks to camcorders or high-speed cameras. Traditionally, 2D-DIC operates on image pairs $[9,10,11]$, and hence a long temporal series is of little use. On the contrary, if the overall displacement over the entire time sequence is large, one may have to break the analysis into time intervals which are finally "chained" to obtain the entire displacement field. When updating the reference picture [12], this procedure involves cumulative errors that are prejudicial to the displacement uncertainty.

The principle of the proposed approach is to extend to the time domain the regularization strategy used spatially. If the velocity field evolves smoothly in time, the above discussion about the $\beta$ parameter may be readily applicable to 
include the time dimension. Thus, for the same $\beta$ value, small elements along the space direction(s) may still offer a good accuracy provided a sufficient number of images is considered along the time axis for each element. This temporal series may be used to compensate for the poor quality of each individual image.

Some approaches post-process a posteriori the measured velocity fields to extract, say, the coherent part of the latter [13] or to filter the measured data [14]. The objective of the present work is to propose an a priori approach in which a space-time decomposition is sought. The main advantage of the proposed method is the large number of pictures used that may allow one to reach the same uncertainty level with a small amount of spatial information, counterbalanced by a large amount of temporal data.

Sequences of images can be obtained from standard movies. To benefit from the large number of images, a temporal regularization is called for. For instance, one may seek for a steady-state velocity fields [15], or in the present case velocity fields that are decomposed over a set of piece-wise linear fields in space and time. This type of description is developed in the same spirit as global approaches $[16,17]$, and in particular to finite-element based correlation algorithms whereby the displacement field is described by finite element shape functions of the space variables $[8,18]$.

Along those lines different strategies can be considered. The direct transposition of DIC is to search for displacement fields in space and time simultaneously. This route is not followed here since the sought fields are velocities and strain rates. It is well known that (time or space) derivatives will increase the noise level, and thus the displacement-formulated strategy may reveal unreliable. Therefore, the choice was made to focus directly on the velocity field as the main unknown to the problem. It will be shown that in spite of the fact that this velocity is the time derivative of displacement, good performances will be reached.

In the present case, a 2D approach is developed, namely, $1 \mathrm{D}$ in space and 1D in time. It is referred to as DIC applied to analyze movies (or MovieDIC). The paper is organized as follows. First, the principle of the method is 
described. Then, artificial pictures are generated and the technique is carried out to determine a priori performances. Last, the spatiotemporal approach is applied to analyze the kinematics of a sample in a split Hopkinson pressure bar test.

\section{Principle of the spatiotemporal analysis}

The first step of the analysis consists in creating the so-called spatiotemporal map. For each picture, where $x, y$ are the image coordinates, taken at several instants of time $t$, the gray level for a particular (chosen) position $(x, y)$ is represented as a function of time $t$. Therefore, for a fixed $y$ coordinate a sequence of images becomes an $f(x, t)$ map. The stacking principle is depicted in Figure 1. The restriction to a single spatial coordinate ( $y$ being fixed) is suited for problems where the velocity is essentially along the $x$ axis.

The measurement technique is based upon the conservation of the brightness $[19,20]$. The advection of the texture by a velocity field $v$ (along the $x$-axis) is expressed as

$$
f(x+v \mathrm{~d} t, t+\mathrm{d} t)=f(x, t)
$$

where the increment $\mathrm{d} t$ corresponds to one time interval between successive images (i.e. a "time pixel"). The aim is to estimate the velocity field $v(x, t)$ by using the brightness conservation. Minimization of the quadratic difference $\tau$ over space and time is used

$$
\tau=\int_{x} \int_{t}[f(x, t)-f(x+v(x, t) \mathrm{d} t, t+\mathrm{d} t)]^{2} \mathrm{~d} x \mathrm{~d} t
$$

The velocity field is decomposed over a basis of functions $\phi$ and $\varphi$ as follows

$$
v(x, t)=\sum_{i, j} a_{i j} \phi_{i}(x) \varphi_{j}(t)
$$

In the present case, finite-element shape functions are chosen, and their simplest form is adopted, namely, a piece-wise bi-linear description of the velocity field. However, it is conceivable to consider other sets of either continuous functions [15] or even discontinuous functions [21, 22]. 
The proposed scheme is to solve this non-linear problem iteratively by a progressive adjustment of the velocity to the tangent linearized problem. The initialization of the unknown velocity field is here chosen to be equal to zero, $v^{0}(x, t)=0$. However, if a predetermination of the velocity field is available, it is straightforward to include it at this stage. The velocity $v^{(n+1)}(x, t)$ at step $n+1$ of this iterative scheme is determined from the Taylor expansion of the objective functional

$$
\tau=\int_{x} \int_{t}\left[f\left(x^{(n)}, t-\mathrm{d} t\right)-f_{, x}(x, t)\left(v^{(n+1)}-v^{(n)}\right) \mathrm{d} t-f(x, t)\right]^{2} \mathrm{~d} x \mathrm{~d} t
$$

with $f_{, x}(x, t)=\partial f(x, t) / \partial x$. In this expression $x^{(n)}$ is a short hand notation for the value $x^{\prime}$ such that $x^{\prime}+v^{(n)}\left(x^{\prime}, t-\mathrm{d} t\right) \mathrm{d} t=x$. Note that the above expression is a specific choice out of many equivalent ones that differ only through second order terms. The advantage of this particular form is that the correction field is multiplied by $f_{, x}(x, t)$, which may be computed once for all iterations. This will ease the computational work as shown in the following.

One difficulty of the above approach, in particular for low quality images, is the use of a space derivative that may render the procedure sensitive to noise. A filtering of the images may be used. Note that in this case, the band filtering used in space and time should be adjusted so that their bounds are in proportion of the mean velocity.

The decomposition (3) is introduced in Equation (4) and minimization with respect to $a_{i j}$ leads to a linear system

$$
\begin{aligned}
& \sum_{i, j}\left(\int_{x} \int_{t}\left[\phi_{i}(x) \phi_{k}(x) \varphi_{j}(t) \varphi_{l}(t) f_{, x}^{2}(x, t)\right] \mathrm{d} x \mathrm{~d} t\right) a_{i j}^{(n+1)} \\
& =\sum_{i, j}\left(\int_{x} \int_{t}\left[\phi_{i}(x) \phi_{k}(x) \varphi_{j}(t) \varphi_{l}(t) f_{, x}^{2}(x, t)\right] \mathrm{d} x \mathrm{~d} t\right) a_{i j}^{(n)} \\
& +\int_{x} \int_{t}\left(\phi_{k}(x) \varphi_{l}(t) f_{, x}(x, t)\left(f\left(x^{(n)}, t-\mathrm{d} t\right)-f(x, t)\right)\right) \mathrm{d} x \mathrm{~d} t
\end{aligned}
$$

This elementary step is written in compact form as

$$
M_{i j k l} a_{i j}^{(n+1)}=B_{k l}^{(n)}
$$

The reason for the specific choice made in Equation (4) is now clear, namely, matrix $\mathbf{M}$ is computed once for all at the first iteration, and it does not depend 
on the current evaluation of the velocity field. However the second member, $\mathbf{B}$ is dependent on $v^{(n)}$, but its evaluation is much less demanding computationally than M. At each step, the "deformed" image $f\left(x+v^{(n)} \mathrm{d} t, t+\mathrm{d} t\right)$ is corrected by using the velocity field estimate at the previous step in order to compute the second member. By inverting (6), the unknown degrees of freedom $a_{i j}^{(n+1)}$ are obtained, and thus the corresponding velocity field is estimated. Convergence, based on a measure of the norm of $a^{(n+1)}-a^{(n)}$, is reached in a few iterations (typically less than 10). By integrating the velocity field with respect to time, the displacement and thereafter the strain fields are obtained.

In order to validate the approach, the objective function is considered. Its value, normalized by the image size $\left(n_{x} \times n_{t}\right)$,

$$
R=\sqrt{\tau /\left(n_{x} n_{t}\right)}
$$

gives the mean gray level difference of the matching of $f(x, t)$ with $f(x, t+\mathrm{d} t)$ using the measured velocity field. It is thus a global measure of the quality. Moreover, because $\tau$ is a space-time integral of the square of a residual field,

$$
\delta \equiv \frac{1}{\Delta}\left|f(x, t)-v(x, t) \mathrm{d} t f_{, x}(x, t)-f(x, t+\mathrm{d} t)\right|
$$

which gives the local contribution of each pixel to the global residual. To make this density dimensionless, it is rescaled by the dynamic range of the original image line $\Delta=\max [f(., t=0)]-\min [f(., t=0)]$.

The algorithm will be checked below against test cases for which the velocity field will be known exactly. In those cases, two quality indicators are introduced, namely, the systematic error $\eta$ and the corresponding standard uncertainty $\sigma$

$$
\begin{aligned}
\eta & =\frac{1}{n_{x} n_{t}} \sum_{i=1}^{n_{x}} \sum_{j=1}^{n_{t}}\left(A_{i j}^{\text {pre }}-A_{i j}^{\text {meas }}\right) \\
\sigma & =\sqrt{\frac{1}{n_{x} n_{t}} \sum_{i=1}^{n_{x}} \sum_{j=1}^{n_{t}}\left(A_{i j}^{\text {pre }}-A_{i j}^{\text {meas }}\right)^{2}}
\end{aligned}
$$

where $A_{\text {pre }}$ is the prescribed value and $A_{\text {meas }}$ its measured counterpart. The quantities $A_{i}$ will denote velocity, displacement, strain or strain rate data. 
The directly measured quantity is the velocity field $v$. From the latter, the longitudinal strain rate $D_{x x}$ is computed as

$$
D_{x x}(x, t)=\frac{\partial v}{\partial x}(x, t)
$$

which, from the present choice of basis for the velocity (i.e., linear in space and time), is a piecewise constant function in $x$, and piecewise linear and continuous in time. The derivation is performed by centered finite differences.

From the velocity field it is also possible to compute the displacement field (trajectories) from an explicit time integration, and sub-pixel linear interpolation of the velocity, which is an exact result because of the choice of the shape function

$$
x(t)=x_{0}(t=0)+\int_{0}^{t} v\left(x\left(t^{\prime}\right), t^{\prime}\right) \mathrm{d} t^{\prime}
$$

The velocity field is computed on the entire space-time domain. In the sequel, in order to use a Fourier-based filtering of the spatio-temporal image, it is useful to extrapolate the observed domain to a larger domain. For instance elements that are not present over the space interval at the initial time, $t_{0}$, may enter the observed scene at a later time, $t_{1}$. In this case, when needed, we assume that a constant velocity was followed in the time interval $\left[t_{0} ; t_{1}\right]$. This conventional procedure allows to minimize edge effects for the Fourier filtering, yet it is to be underlined that the extrapolated domain is removed after filtering, and hence this ad hoc extrapolation procedure has a very low impact on the measurement over the observed scene. If instead a zero padding is used, edge effects are observed to be detrimental to the quality of the determined velocity field.

The corresponding longitudinal strain $\epsilon_{x x}$ is expressed as

$$
\epsilon_{x x}(x, t)=\frac{\partial u}{\partial x}(x, t)
$$

The derivation is carried out by a centered finite differences scheme.

\section{A priori analyses}

The three maps shown in Figure 2 are artificially generated so that the correlation length of the first line was approximately equal to 3 pixels. The 
size of each map is equal to $256 \times 256$ pixels because a wide range of mesh sizes $2^{i}$ pixels $(i=3 \ldots 7$ along the time dimension, and $i=1 \ldots 7$ along the space dimension) are used, with a reasonable computation time (e.g., $8 \times 8$-pixel elements require less than twenty seconds of computation time on a standard PC). Moreover, small mesh sizes along the time dimension are not considered except for the discontinuous map because in practice the aim of this method is to use many time steps to compensate for the small amount of information along the space dimension.

As mentioned in Section 2, images may be filtered in Fourier space, but this operation has its principal axes along the space and time directions, and hence it is unsuited to the spatio-temporal maps. The chosen strategy was first to correct this map. The evaluation of the velocity field allows for the trajectories to be determined, and hence for each point $(x, t)$ one may trace back the trajectory going through this point up to the origin of time $\left(x_{0}(x, t), 0\right)$. The "corrected" map $g(x, t)$ is built so that $g\left(x_{0}(x, t), t\right)=f(x, t)$. By hypothesis, $g(x, t)$ should be time invariant (and equal to $f(x, 0)$ ). In this transformation, the boundary of the domain becomes more or less lozenge shaped (a lozenge would be obtained for a uniform and constant velocity field). This corrected map $g$ is embedded in a larger rectangle and the missing information is completed using (conventionally) a constant velocity as explained in the previous section. A low pass Gaussian filter is applied over the $g$ field and the inverse transformation $g \rightarrow f$ is applied to restore back the original domain shape. The extrapolated data is thus removed. The standard deviation of the Gaussian filter is equal to 1 pixel for all the studied cases.

\subsection{Constant velocity}

In this first case, the prescribed velocity is constant and equal to $(1+\sqrt{5}) / 4 \approx$ 0.81 pixel per image. This specific number, half the golden mean, is chosen because it induces sub-pixel components of the displacements that are close to a uniform distribution (i.e., the standard deviation of the sub-pixel component distribution is 0.290 to be compared with $1 / \sqrt{12} \approx 0.289$ for a uniform dis- 
tribution). The spatiotemporal map corresponding to this case is shown in Figure 2(a). In the present case a bilinear gray level interpolation is used. Further, it is worth noting that the measurement basis contains the studied velocity field.

The present case is a typical baseline analysis in classical DIC techniques [23, 8]. It allows one to check the implemented algorithms. Let us first consider the velocity field. The only parameters are the sizes $\ell_{x}$ and $\ell_{t}$ of the spatial and temporal discretizations. The systematic error and the standard uncertainty are shown in Figure 3(a) and Figure 3(b). A decrease of the standard uncertainty is observed with increasing element sizes, be it spatial or temporal. The larger the element, the larger the number of data (i.e., pixels), the more accurate the measured velocity. Differences between the prescribed and measured velocity fields for $8 \times 8$-pixel elements are shown in Figure 3(c).

The second studied quantity is the displacement field for which the systematic error and the standard uncertainty are given in Figure 4(a) and Figure 4(b). A decrease of the uncertainty is observed when the element size increases, while the systematic error is approximately constant. Differences between the prescribed and measured displacement fields are shown in Figure 4(c) for the largest element size, $128 \times 128$-pixel. The shape of the map is due to the fact that the displacement of the first line of the map is followed. The values of the displacement field are ranging from 0 and 256 pixels, whereas the velocity level is close to 0.809 pixel per image. The values of the measured displacement fields are in good agreement with the prescribed ones.

A good way of estimating the quality of the displacement field determination is to construct the corrected spatiotemporal map, $g$, where the effect of the estimated velocity is removed (the one used for the Fourier filtering). The result is shown in Figure 4(d) for a small element size, $8 \times 8$-pixel. For larger element size, no difference can be perceived with bare eyes. The spatial position is corrected from the beginning to the end. The particular shape of the map is caused by the fact that there is no information outside the bounds of the image. Therefore, the repositioning cannot be performed at these locations. 
For real applications, the actual velocity field is unknown. Thus the quality evaluation that is used is the residual, $f(x, t)-f(x+v(x, t) \mathrm{d} t, t+\mathrm{d} t)$, computed from the estimated velocity field. Figure 5(a) shows the normalized mean residual for different element sizes and Figure 5(b) the residual map for a particular element size $(8 \times 8$ pixels $)$. In that case, the residuals are virtually constant for all element sizes, and the higher values are located close to the left edge of the map, presumably because of an imperfect extrapolation procedure. It is to be noted that edges are always a weak point in DIC analyses based on a similar methodology.

In many situations, the end user of the measurements is more interested in strain and/or strain rate fields than in the displacement or velocity fields. The latter are interpreted in terms of mechanical behavior, whereas the former include rigid body components that are usually not useful to understand or capture the true mechanical response of a system under investigation. However, the strain or strain rate fields require spatial derivations that amplify the noise level. The systematic error and standard uncertainty of the strain fields are shown in Figure 6(a) and Figure 6(b). In this particular case, the prescribed strain field is equal to zero. Figure 6(c) shows the difference between the prescribed and measured strain fields for elements of size $8 \times 8$ pixels. This field is similar to the displacement field and the effect of derivation is observed.

In order to validate the benefit of the proposed approach with respect to classical tools, the test case with a constant velocity field is an appropriate example. A classical tool would ignore the time dimension and thus it consists in analyzing two consecutive lines. These lines are partitioned into intervals (i.e., "Zone Of Interest", or ZOI), over which the mean displacement is searched for, independently for each interval. It is to be emphasized that such a "classical" tool is seldom used in one space dimension. However, there is no limitation in this respect. No convergence was obtained for ZOI sizes smaller than 8 pixels. For this size ( $\ell_{x}=8$ pixels) up to 32 pixels, the displacement field uncertainty decreased from 0.023 to 0.006 pixel. This uncertainty is always worse than the one obtained in the spatio-temporal framework. 
A global one dimensional DIC approach is also performed. It is based on a continuous kinematic basis (linearly varying displacement field) over a partition of the line into 1D-elements, or intervals as in the previous case. This treatment is similar to the spatio-temporal approach at the exception of the incorporation of time in the kinematics. The prescription of a continuous displacement (here equivalent to velocity since only two consecutive lines are considered) field helped significantly the convergence since elements as small a size of 3 pixels could be handled without convergence problems. For $\ell_{x}=4$ pixels, the uncertainty was observed to amount to 0.042 pixel. In the same range as above, from $\ell_{x}=8$ to 32 pixels, the uncertainty decreased from 0.020 down to 0.005 pixel. Thus continuity revealed useful to reduce the uncertainty level, yet at a level higher than the proposed spatio-temporal approach.

As a last comparison with classical approaches, the maximum displacement that would allow for convergence is evaluated. It was found that for velocities as large as about 1.5 times the correlation length, the computation converged. This allows one to consider velocities as large as 6 pixels per time step. Being able to handle such large displacements without any special initialization is another benefit of the proposed method.

To summarize, the time regularization that is proposed herein allows one to extend considerably the range of convergence of the DIC algorithm. Moreover, it reduces the uncertainty level. Note that the rather large level of uncertainty as compared to traditional DIC is due to the fact that in one dimension the number of pixels used to determine a kinematic degree of freedom is small compared with 2D approaches.

\subsection{Parabolic velocity field}

The expression of the velocity field in this second test case reads

$$
u=\frac{a t^{2} x^{2}}{256^{2}}
$$

with $a=(1+\sqrt{5}) / 4 \approx 0.81$ pixel per image. In that case, the degree of the prescribed velocity is higher than that of the measurement basis. Thus 
systematic bias due to the projector error on the discretization basis is expected to penalize large element discretizations.

Figure 2(b) shows the spatiotemporal map corresponding to the chosen velocity field. First, the systematic error and the standard uncertainty for the measured velocity field are analyzed as functions of the element size in Figure 7(a) and Figure 7(b). When the discretization becomes too crude, the two quantities increase because the measurement basis is not rich enough to capture a parabolic field. For instance, for an element size equal to $128 \times 128$ pixels (Figure $7(\mathrm{~d})$ ), the difference between the prescribed and measured velocity fields is less satisfactory than for sizes $32 \times 32$ pixels (Figure $7(\mathrm{c})$ ) even though fewer degrees of freedom are measured in the first case.

Moreover, the difference clearly shows the underlying mesh (made of four elements in the first case), and its maximum is located on the edges and in the middle of the elements. However, even for a large element size, the map is reconstructed accurately as can be seen on Figure 8(c). Although the description of the velocity is poor, the mean trend is well captured. The gray level residuals as functions of the element size are shown in Figure 8(a) and the difference between the measured (with $32 \times 32$-pixel elements) and prescribed trajectories are shown in Figure 8(b). In this case too, the residuals are approximatively identical for tested element sizes. Figure 9(a) and Figure 9(b) show the change of the strain error and uncertainty for different element sizes. The optimum size is observed to be $32 \times 32$ pixels in the present case. (Note however that this optimum size is dependent on the observed velocity field.)

\subsection{Discontinuous velocity}

In this last synthetic example, the prescribed velocity field is discontinuous. For times 1 to 100 , and 141 to 256, the velocity is equal to 0 . In between, the velocity is constant and equal to $v=(1+\sqrt{5}) / 4$ pixel per image. The spatiotemporal map is shown in Figure 2(c).

The same analysis as previously shown is carried out. However, the conclusions are not the same. The change of the systematic error and the standard 
uncertainty (Figure 10(a) and Figure 10(b)) for the displacement fields with the element size shows that both quantities reach a minimum for a small spatial size, typically $2 \times 32$ pixels or $4 \times 16$ pixels, along the direction transverse to the discontinuity.

Moreover, the differences shown in Figure 10(c) for the displacement fields provide an additional information because they give exactly the position and intensity of the perturbations associated with the chosen measurement basis. From Figure 10(c) it is concluded that the error is the most important exactly where the discontinuity is located and not near the edges.

The gray level residuals are shown in Figure 11(a). The residual decreases when the element size along the time dimension decreases too. The minimum value is reached for a size equal to $4 \times 128$ pixels. Over the discontinuity line, the difference between the prescribed and measured displacement leads to a systematic error, which is partially corrected by the second discontinuity seen in the corrected image. However, this incomplete correction induces a nonvanishing strain field, which in reality does not exist.

Unlike the other tested maps, the image is not completely corrected by using the displacement field (Figure 11(b)). A solution is to use enriched shape functions as in eXtended Finite Element techniques [24]. This second solution is implemented in DIC techniques when only two pictures are analyzed [22].

\section{Application to a tensile test}

In this last part, a real experimental case is studied. A cylinder-shaped tantalum specimen is subjected to a tensile test in split Hopkinson pressure bars $[25,26,27,28]$. In the present case, out-of-plane displacements remain small so that no correction procedure is used [29]. The spatiotemporal map is shown in Figure 1, whose size is $201 \times 351$ pixels in space and time, respectively. A pixel represents a size equal to $165 \mu \mathrm{m}$ and the frame rate is equal to 30,000 fps.

The sample is first subjected to a tensile pulse followed by a quiescent period when the loading wave has traveled out of the specimen. Wave reflection at the 
end of the bar, leads to a second tensile pulse episode. During the second pulse, failure occurs through a localized necking instability.

First, at a given position (Figure 1), the corresponding velocity, displacement and strain maps are given in Figures 12(a), 12(b) and 12(c), respectively, for $4 \times 64$-pixel elements. This size is chosen by using the results of Section 3.3. The present approach is also compared with a Q4-DIC code in which a spatial piece-wise bilinear (Q4 finite element) kinematics is implemented [8]. In that case, a series of 2D displacement fields are obtained for different instances of time. For the spatiotemporal analysis, 40 maps are generated for the tantalum sample corresponding to several vertical positions. For each of them, a computation is carried out independently. The displacement field for image no. 35 is computed by using both techniques as shown in Figure 13(a). A good alignment of the camera with the sample axis allows for the present analysis. In terms of transverse displacements that may violate the 1D-approach, they were measured with Q4-DIC. The average transverse displacement is estimated to amount to about 0.02 pixel, and hence a 1D approach is legitimate. More precisely, the displacement field given by Q4-DIC is the average of the lines where it is measurable (i.e., over 18 pixels). For the spatiotemporal approach, it is exactly the same operation, although an interpolation is not needed since the displacement field is directly computed for each line. A good agreement between the two techniques is observed. Beyond position 350 pixels, noise is observed because of the small element size (i.e., $6 \times 6$ pixels) chosen for the Q4-DIC approach. Another advantage of the spatiotemporal approach is its tolerance to large values of the displacement field.

When cumulated, a small velocity level may eventually induce large displacements that are difficult to estimate by using standard DIC techniques. This explains why the displacement field for the image corresponding to time no. 175 is measurable with the spatiotemporal approach (Figure 13(b)). For this picture, necking is very important and the engineering strain field is more suited to see the exact position and intensity of this phenomenon. Moreover, even if the result is shown for a given time, it is possible to obtain the same field 
at different times and consequently to follow the growth of necking. This result shows that localized phenomena can be captured with a spatial resolution that is smaller than that allowed by classical 2D-DIC techniques [30, 31, 32].

To compare the present results with previous studies, gray level residuals are shown in Figure 14(a), for different element sizes, and the difference between the computed and measured trajectories is given in Figure 14(b). The levels of the residuals are higher than those for the discontinuous velocity field. This is partly due to the acquisition noise of the images, and presumably also to the second discontinuity along the space dimension. However, even though higher than in artificial cases, the overall level is sufficiently low to allow us to deem the present results trustworthy.

\section{Conclusion}

A novel approach was developed to determine velocity fields based on the global registration of a series of digital images. The velocity field is decomposed onto a basis of continuous functions using Q4P1-shape spatiotemporal functions. Displacement, strain and strain rate fields are subsequently estimated.

The performance of the algorithm is tested on several test maps in order to evaluate the reliability of the estimation, which is shown to allow for either an excellent accuracy for continuous velocity fields, or reasonable estimates for discontinuous velocities. In the latter case, it is shown that the space/time discretization has to be adapted to properly capture the specific features of the velocity field. Last, this method is used to analyze a tantalum specimen subjected to a tensile test in Hopkinson bars where the performance of the technique is compared with a finite element based DIC approach. A good agreement between both techniques is observed.

The space-time approach is particularly suited to experiments in which small resolution pictures obtained by, say, camcorders or high speed cameras, yield a large amount of images. This is for instance the case of split Hopkinson pressure bar experiments. The time regularization proposed herein enables for the use of fine spatial discretizations with reasonable uncertainty levels to capture localized 
phenomena such as necking. It is an alternative to local 2D-DIC approaches [30, 31, 32] and an extension of Q4-DIC [8]. Discontinuous enrichments such as those proposed for a Q4-DIC scheme [33] may be added in the future.

The generalization to 2D spatial discretizations, or 3D discretizations coupled with 1D time discretizations is currently investigated. The output will then be 3D and 4D velocity fields that can be used, for instance, to identify or validate the parameters of constitutive equations.

\section{Acknowledgements}

The authors wish to thank Nicolas Granier of CEA Valduc for providing the pictures of the experiment analyzed herein.

\section{References}

[1] S. Avril, M. Bonnet, A.-S. Bretelle, M. Grédiac, F. Hild, P. Ienny, F. Latourte, D. Lemosse, S. Pagano, E. Pagnacco and F. Pierron, "Overview of identification methods of mechanical parameters based on full-field measurements," Exp. Mech. 48, [4], 381-402 (2008).

[2] P. K. Rastogi, edt.,Photomechanics, (Springer, Berlin (Germany), 2000), 77.

[3] I. Chasiotis (edt.), "Special issue on nanoscale measurements in Mechanics," Exp. Mech. 47, [1], (2007).

[4] K. Han, M. Ciccotti and S. Roux, Measuring Nanoscale Stress Intensity Factors with an Atomic Force Microscope, EuroPhys. Lett. 89 [6] (2010) 66003.

[5] M. A. Sutton, J.-J. Orteu and H. Schreier, Image correlation for shape, motion and deformation measurements: Basic Concepts, Theory and Applications, (Springer, New York, NY (USA), 2009).

[6] M. Küntz, M. Jolin, J. Bastien, F. Perez and F. Hild, "Digital image correlation analysis of crack behavior in a reinforced concrete beam during a load test," Canad. J. Civil Eng. 33, 1418-1425 (2006). 
[7] S. Bergonnier, F. Hild and S. Roux, "Digital image correlation used for mechanical tests on crimped glass wool samples," J. Strain Analysis 40, 185-197 (2005).

[8] G. Besnard, F. Hild and S. Roux, "Finite-element displacement fields analysis from digital images: Application to Portevin-Le Châtelier bands," Exp. Mech. 46, 789-803 (2006).

[9] P. J. Burt, C. Yen and X. Xu, Local correlation measures for motion analysis: a comparative study, Proceedings IEEE Conf. on Pattern Recognition and Image Processing, (1982), 269-274.

[10] M. A. Sutton, W. J. Wolters, W. H. Peters, W. F. Ranson and S. R. McNeill, Determination of Displacements Using an Improved Digital Correlation Method, Im. Vis. Comp. 1 [3] (1983) 133-139.

[11] M. A. Sutton, S. R. McNeill, J. D. Helm and Y. J. Chao, "Advances in TwoDimensional and Three-Dimensional Computer Vision," in Photomechanics, P. K. Rastogi, edt., (Springer, Berlin (Germany), 2000), 323-372.

[12] F. Hild, B. Raka, M. Baudequin, S. Roux and F. Cantelaube, "Multi-Scale Displacement Field Measurements of Compressed Mineral Wool Samples by Digital Image Correlation," Appl. Optics. IP 41, [32], 6815-6828 (2002).

[13] C. Py, E. de Langre, B. Moulia and P. Hémon, "Measurement of windinduced motion of crop canopies from digital video images," Agric. Forest Meteorol. 130, 223-236 (2005).

[14] L. B. Fore, A. T. Tung, J. R. Buchanan and J. W. Welch, "Nonlinear temporal filtering of time-resolved digital particle image velocimetry data," Exp. Fluids. 39, 22-31 (2005).

[15] S. Bergonnier, F. Hild and S. Roux, "Analyse d'une cinématique stationnaire hétérogène," Revue Comp. Mat. Av. 13, [3], 293-302 (2003). 
[16] E. P. Simoncelli, "Bayesian Multi-Scale Differential Optical Flow," in Handbook of Computer Vision and Applications, B. Jähne, H. Haussecker and P. Geissler, eds., (Academic Press, 1999), pp. 297-422.

[17] B. Wagne, S. Roux and F. Hild, "Spectral Approach to Displacement Evaluation From Image Analysis," Eur. Phys. J. AP. 17, 247-252 (2002).

[18] S. Roux, F. Hild, P. Viot and D. Bernard, "Three dimensional image correlation from X-Ray computed tomography of solid foam," Comp. Part A. 39, [8], 1253-1265 (2008).

[19] C. Fennema and W. Thompson, "Velocity determination in scenes containing several moving objects," Comput. Graph. Im. Proc. 9, 301-315 (1979).

[20] B. K. P. Horn and B. G. Schunck, "Determining optical flow," Artificial Intelligence. 17, 185-203 (1981).

[21] J. Réthoré, S. Roux and F. Hild, "From pictures to extended finite elements: Extended digital image correlation (X-DIC)," C. R. Mecanique. 335, 131137 (2007).

[22] Réthoré, F. Hild and S. Roux, "Shear-band capturing using a multiscale extended digital image correlation technique," Comp. Meth. Appl. Mech. Eng. 196, [49-52], 5016-5030 (2007).

[23] M. A. Sutton, S. R. McNeill, J. Jang and M. Babai, "Effects of subpixel image restoration on digital correlation error estimates," Opt. Eng. 27, [10], 870-877 (1988).

[24] N. Moës, J. Dolbow and T. Belytschko, "A finite element method for crack growth without remeshing," Int. J. Num. Meth. Eng. 46, [1], 133-150 (1999).

[25] D. Hopkinson, "A Method of Measuring the Pressure Produced in the Detonation of High Explosives or by the Impact of Bullets," Phil. Trans. Roy. Soc. A 213437 (1914). 
[26] H. Kolsky, Stress Waves in Solids, (Dover Publications, New York (USA), 1963).

[27] J. Harding, E. O. Wood and J. D. Campbell, "Tensile testing of materials at impact rates of strain," J. Mech. Eng. Sci. 2, 88-96 (1960).

[28] J. E. Field, S. M. Walley, W. G. Proud, H. T. Goldrein and C. R. Siviour, "Review of experimental techniques for high rate deformation and shock studies," Int. J. Impact Eng. 30, 725-775 (2004).

[29] M. A. Sutton, J. H. Yan, V. Tiwari, H. W. Schreier and J.-J. Orteu, "The Effect of Out of Plane Motion on 2D and 3D Digital Image Correlation Measurements," Opt. Lasers Eng. 46 746-757 (2008).

[30] B. Wattrisse, A. Chrysochoos, J. M. Muracciole and M. Némoz-Gaillard, Analysis of strain localisation during tensile test by digital image correlation, Exp. Mech. 41 [1] (2001) 29-39.

[31] P. Schlosser, D. Favier, H. Louche and L. Orgéas, Experimental characterization of NiTi SMAs thermomechanical behaviour using temperature and strain full-field measurements, Adv. Sci. Tech. 59 (2008) 140-149.

[32] S.-H. Tung, M.-H. Shih and J.-C. Kuo, Application of digital image correlation for anisotropic plastic deformation during tension testing, Opt. Lasers Eng. 48 [5] (2010) 636-641.

[33] J. Réthoré, G. Besnard, G. Vivier, F. Hild and S. Roux, Experimental investigation of localized phenomena using Digital Image Correlation, Phil. Mag. 88 [28-29] (2008) 3339-3355. 


\section{List of Figures}

1 Construction of a spatiotemporal map. . . . . . . . . . . . 22

2 Spatiotemporal map corresponding to a constant velocity (a), a parabolic velocity field (b) and a discontinuous velocity field (c).

3 Systematic error (a) and standard uncertainty (b) as functions of the element sizes for the measured velocity field. Difference between the prescribed and measured velocity fields for $8 \times 8$ pixel elements (c). . . . . . . . . . . . . . . . . . . . 2

4 Systematic error (a) and standard uncertainty (b) as functions of the element size for the measured displacement field. Difference between the prescribed and measured displacement fields for $128 \times 128$-pixel elements (c). Reconstruction of the corrected map $g$ by using the measured displacement field for $8 \times 8$-pixel elements $(\mathrm{d}) . \ldots \ldots \ldots \ldots \ldots \ldots \ldots \ldots$

$5 \quad$ Normalized mean gray level residual as a function of the element size for a constant prescribed velocity (a). Residual map between the measured (with $8 \times 8$-pixel elements) and prescribed trajec-

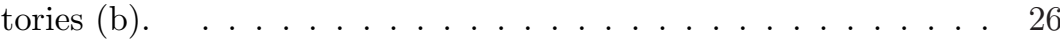

$6 \quad$ Systematic error (a) and standard uncertainty (b) as functions of the element size for the strain field. Difference between the prescribed and measured strain fields for an element of size $8 \times 8$ pixels (c).

$7 \quad$ Systematic error (a) and standard uncertainty (b) as functions of the element size for the measured velocity field when a parabolic velocity is prescribed. Difference between the prescribed and measured velocity fields for $32 \times 32$-pixel elements (c), and $128 \times$ 128-pixel elements (d). 
8 Normalized mean gray level residual as a function of element size for a parabolic prescribed velocity (a). Residual map between the measured (with $32 \times 32$-pixel elements) and the prescribed trajectories (b). Corrected map with the measured displacement field for a $32 \times 32$ pixel element (c). . . . . . . . . . . . . . . . 29

$9 \quad$ Systematic error (a) and standard uncertainty (b) as functions of the element size, for the measured strain field when a parabolic velocity field is prescribed. . . . . . . . . . . . . 30

10 Systematic error (a) and standard uncertainty (b) as functions of the element size for the displacement field when a discontinuous velocity field is prescribed. Difference between the prescribed and measured displacement fields for $8 \times 8$-pixel elements (c). . . . . 31

11 Normalized mean gray level residual as a function of the element size for a discontinuous prescribed velocity (a). Corrected map for $8 \times 8$-pixel elements (b). . . . . . . . . . . . . . 32

12 Velocity (a), displacement (b) and engineering strain (c) maps for the studied test. . . . . . . . . . . . . . . 33

13 Comparison between the displacement field given by the spatiotemporal approach and a Q4-DIC technique (a), the element size is equal to $6 \times 6$ pixels for Q4-DIC and $4 \times 12$ pixels for the spatiotemporal approach. Displacement and engineering strain maps computed with the proposed approach for image no. 175 (just before failure) (b) . . . . . . . . . . . . . . . . . 34

14 Normalized mean gray level residual as function of element size for the real application (a). Difference between the real and reconstructed spatiotemporal maps (b). The latter is reconstructed with the measured velocities $(4 \times 20$-pixel elements $) . \quad \ldots \ldots . \quad 35$ 


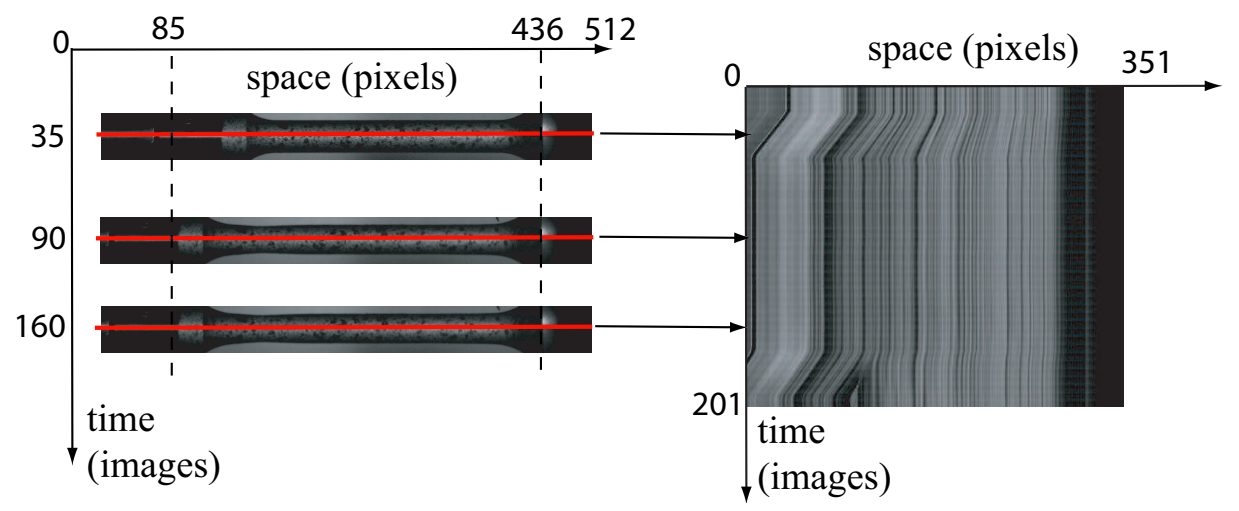

Figure 1: Construction of a spatiotemporal map. 


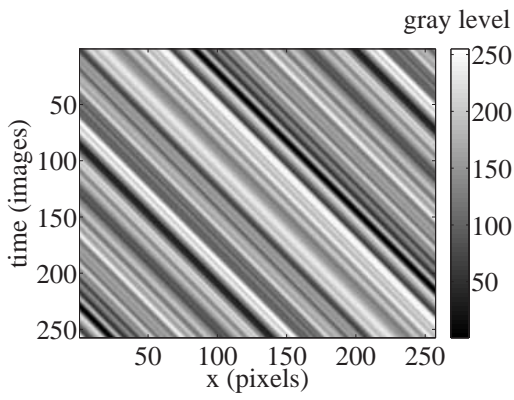

(a)

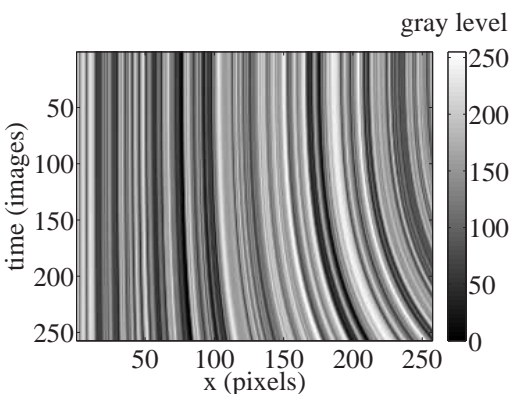

(b)

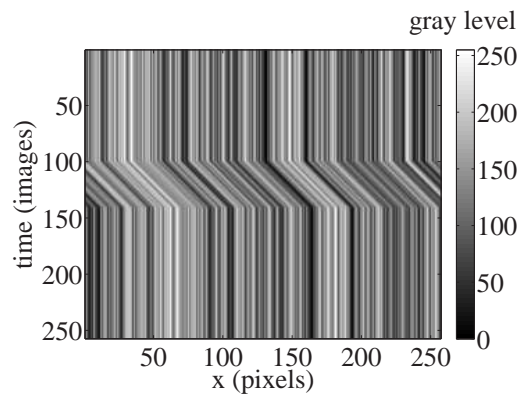

(c)

Figure 2: Spatiotemporal map corresponding to a constant velocity (a), a parabolic velocity field (b) and a discontinuous velocity field (c). 


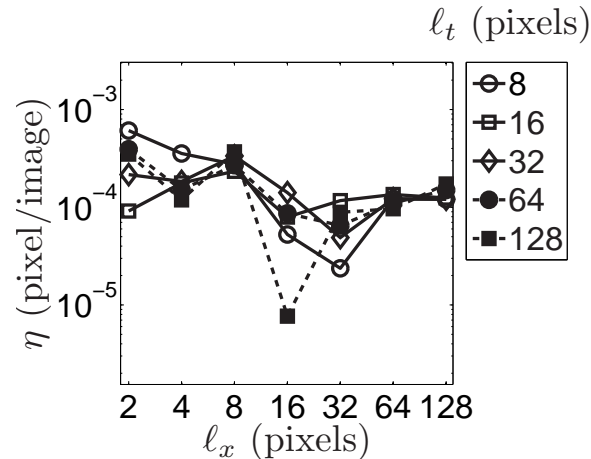

(a)

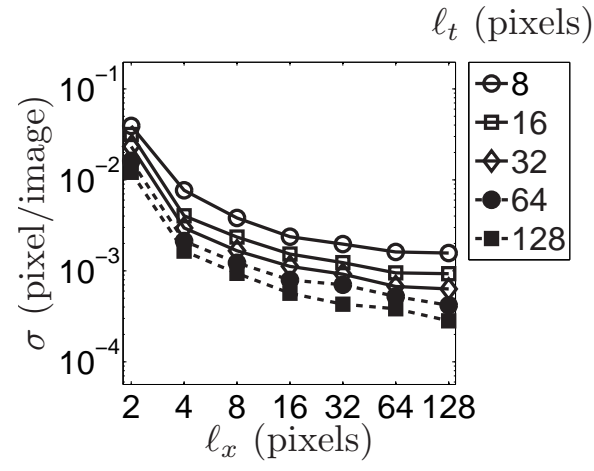

(b)

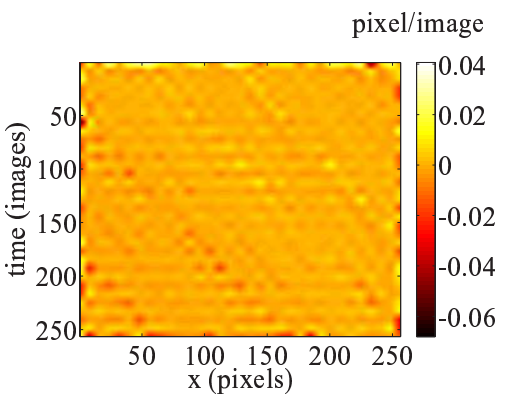

(c)

Figure 3: Systematic error (a) and standard uncertainty (b) as functions of the element sizes for the measured velocity field. Difference between the prescribed and measured velocity fields for $8 \times 8$-pixel elements (c). 


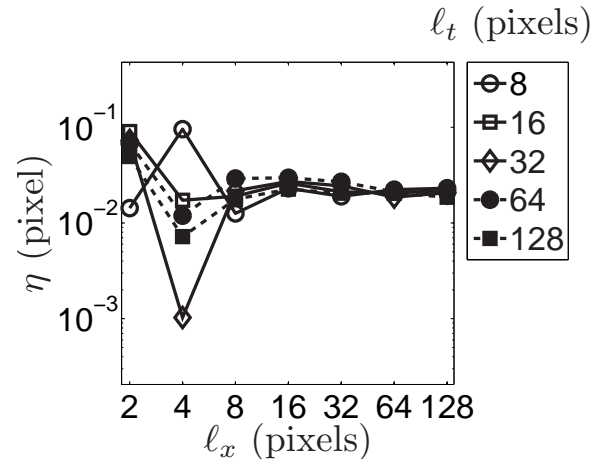

(a)

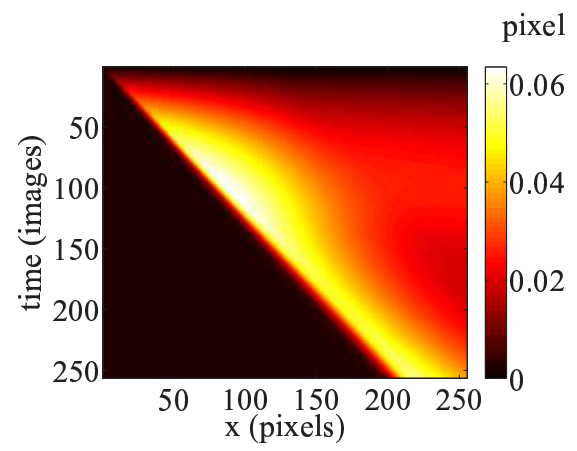

(c)

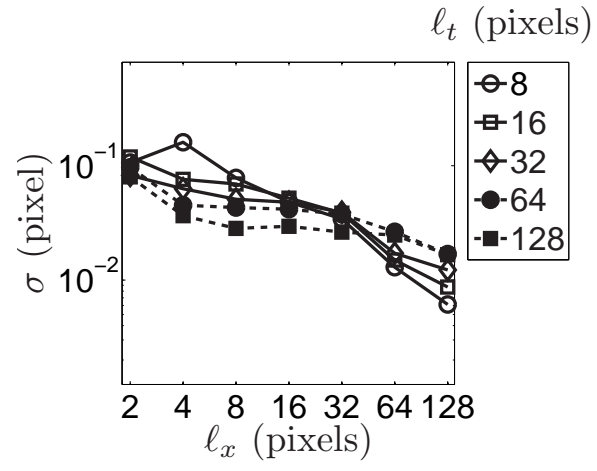

(b)

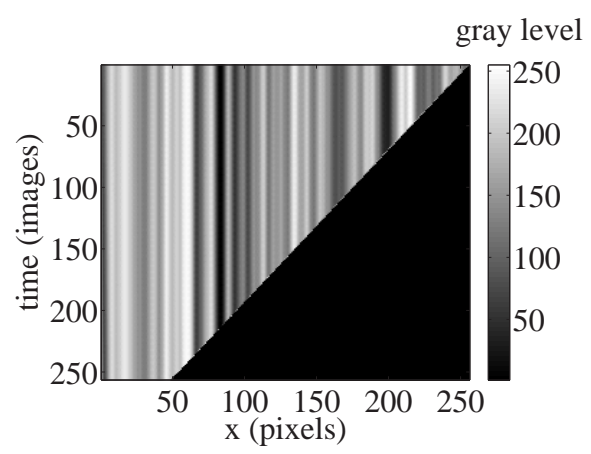

(d)

Figure 4: Systematic error (a) and standard uncertainty (b) as functions of the element size for the measured displacement field. Difference between the prescribed and measured displacement fields for $128 \times 128$-pixel elements (c). Reconstruction of the corrected map $g$ by using the measured displacement field for $8 \times 8$-pixel elements $(d)$. 


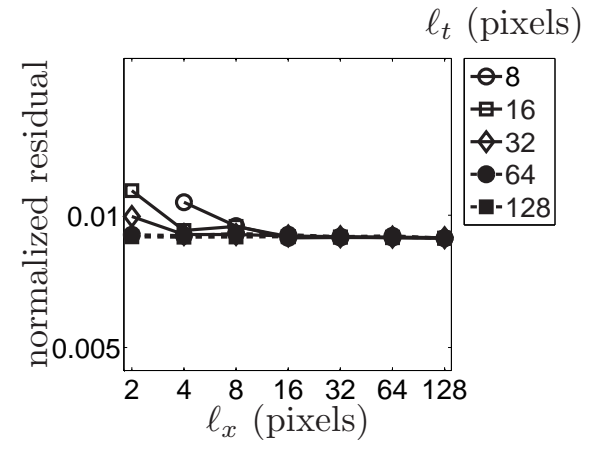

(a)

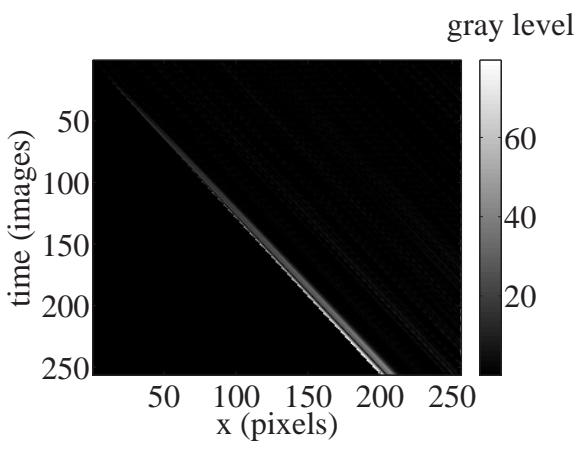

(b)

Figure 5: Normalized mean gray level residual as a function of the element size for a constant prescribed velocity (a). Residual map between the measured (with $8 \times 8$-pixel elements) and prescribed trajectories (b). 


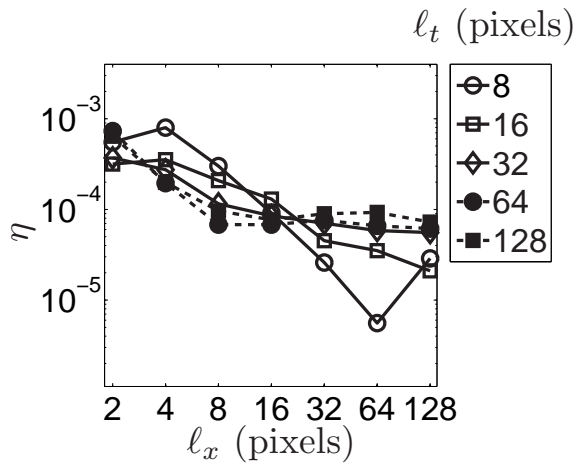

(a)

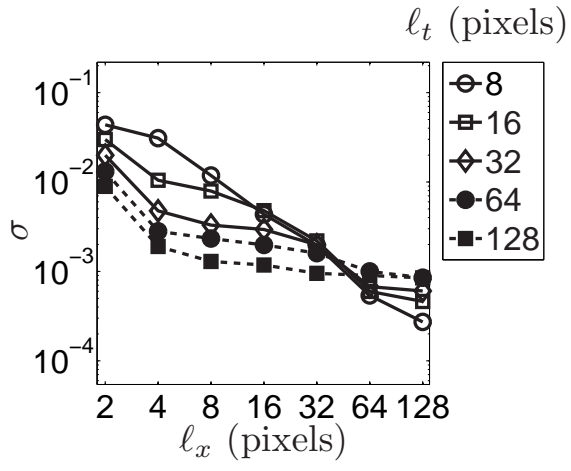

(b)

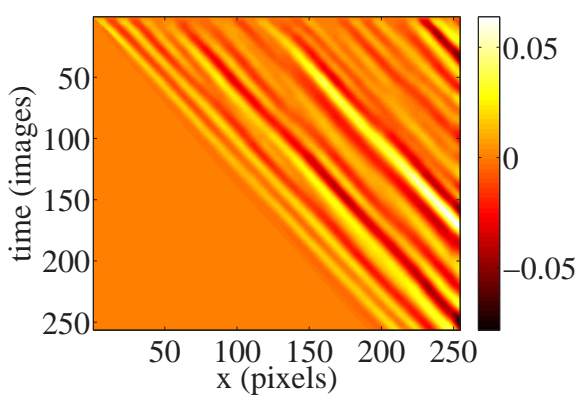

(c)

Figure 6: Systematic error (a) and standard uncertainty (b) as functions of the element size for the strain field. Difference between the prescribed and measured strain fields for an element of size $8 \times 8$ pixels (c). 


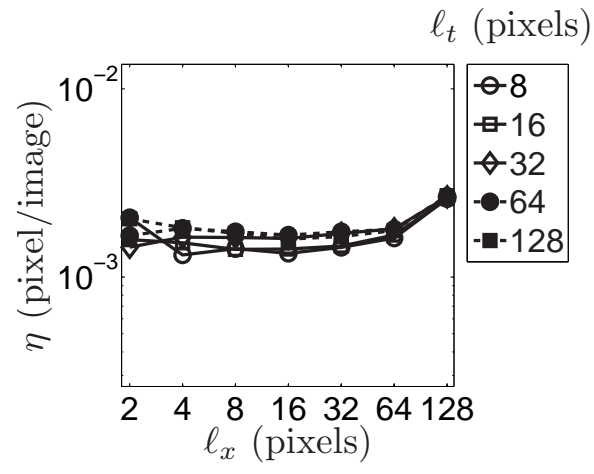

(a)

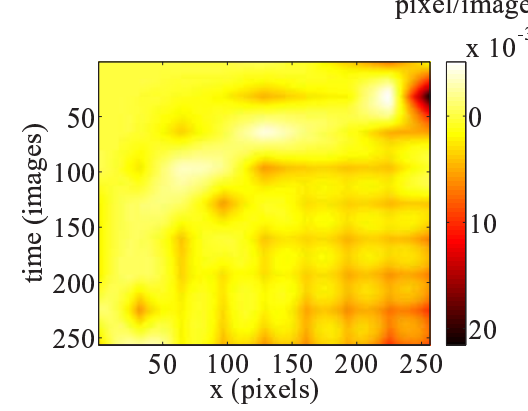

(c)

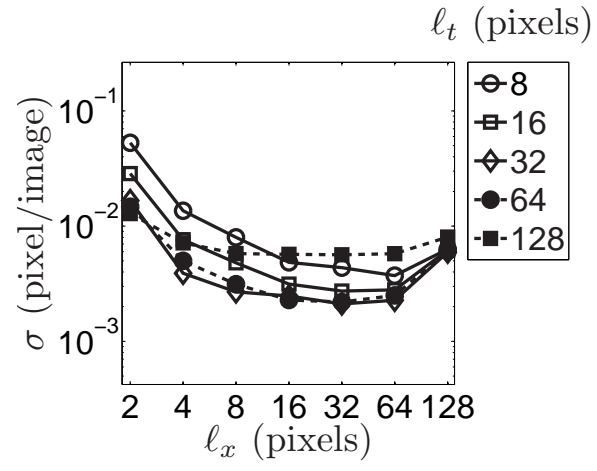

(b)

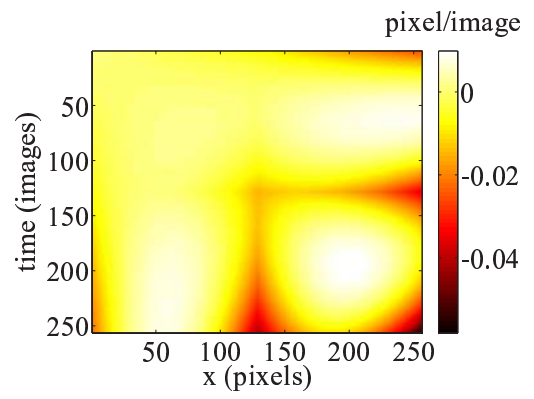

(d)

Figure 7: Systematic error (a) and standard uncertainty (b) as functions of the element size for the measured velocity field when a parabolic velocity is prescribed. Difference between the prescribed and measured velocity fields for $32 \times 32$-pixel elements (c), and $128 \times 128$-pixel elements (d). 


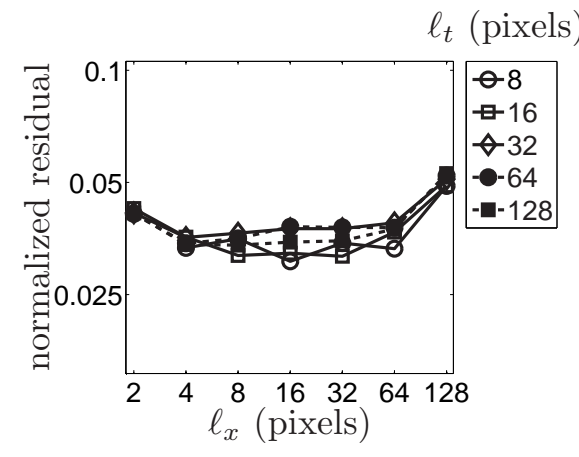

(a)

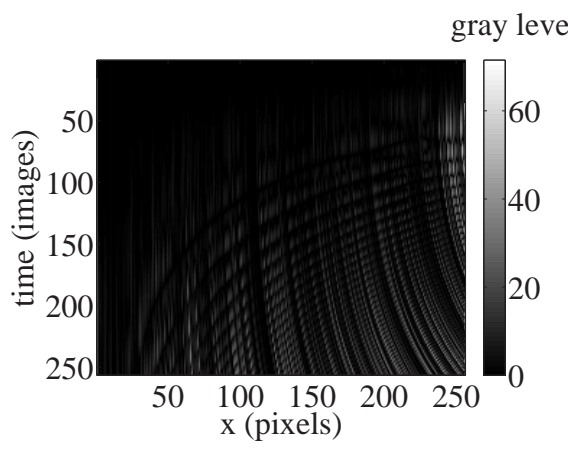

(b)

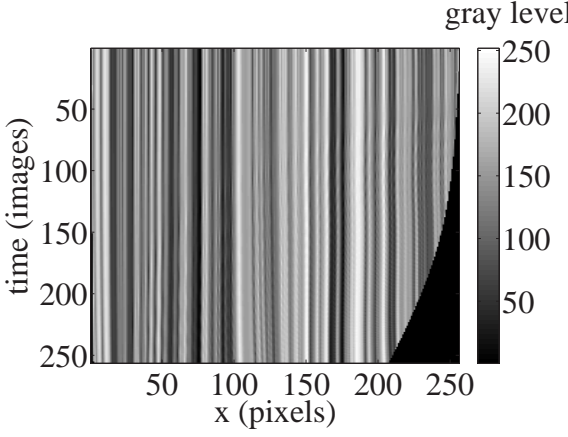

(c)

Figure 8: Normalized mean gray level residual as a function of element size for a parabolic prescribed velocity (a). Residual map between the measured (with $32 \times 32$-pixel elements) and the prescribed trajectories (b). Corrected map with the measured displacement field for a $32 \times 32$ pixel element (c). 


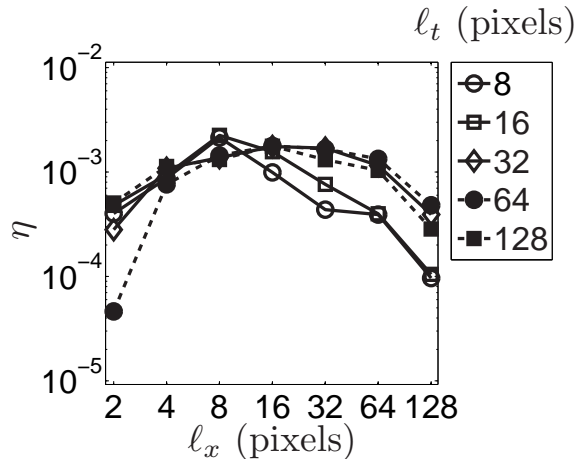

(a)

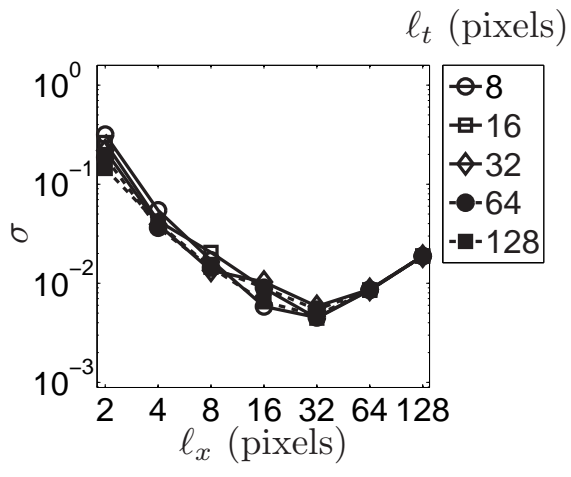

(b)

Figure 9: Systematic error (a) and standard uncertainty (b) as functions of the element size, for the measured strain field when a parabolic velocity field is prescribed. 


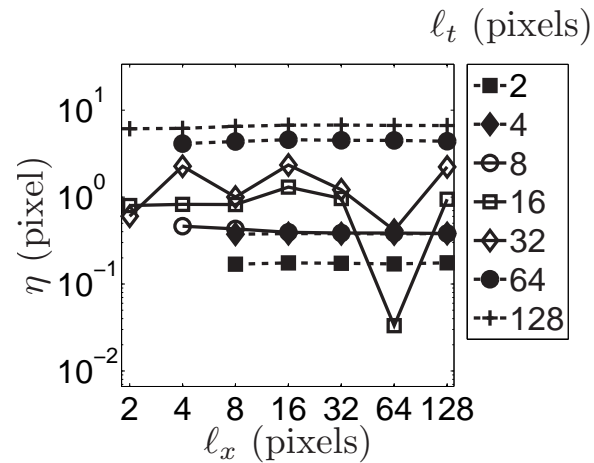

(a)

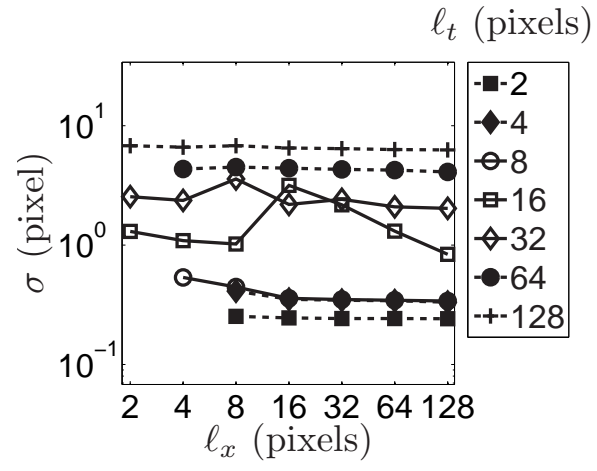

(b)

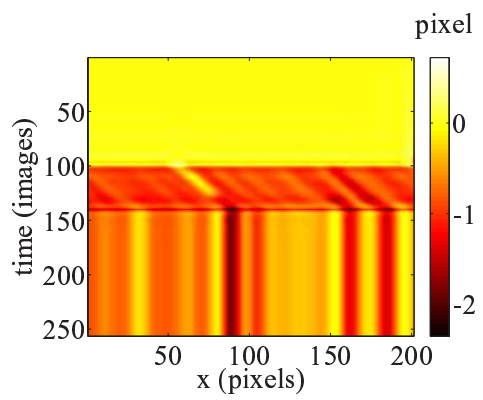

(c)

Figure 10: Systematic error (a) and standard uncertainty (b) as functions of the element size for the displacement field when a discontinuous velocity field is prescribed. Difference between the prescribed and measured displacement fields for $8 \times 8$-pixel elements (c). 


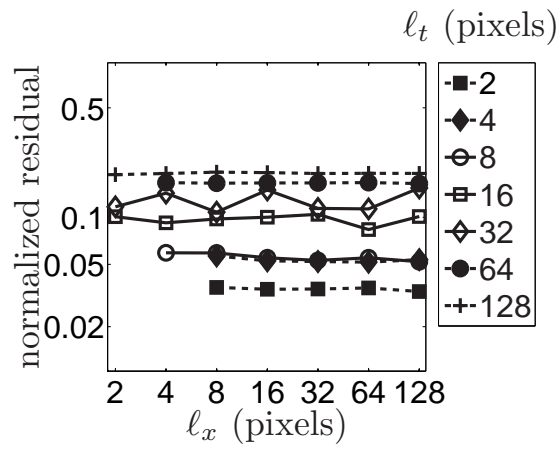

(a)

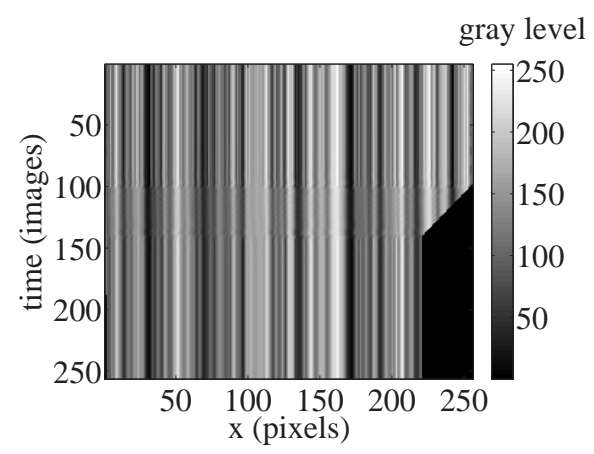

(b)

Figure 11: Normalized mean gray level residual as a function of the element size for a discontinuous prescribed velocity (a). Corrected map for $8 \times 8$-pixel elements (b). 


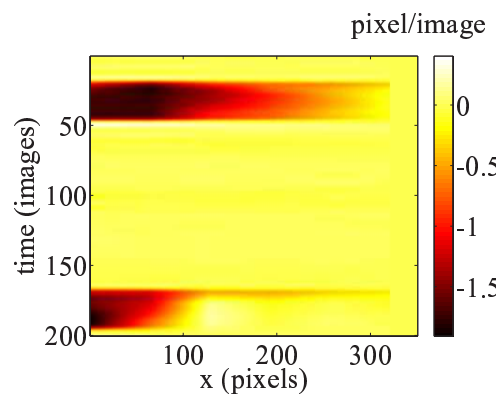

(a)

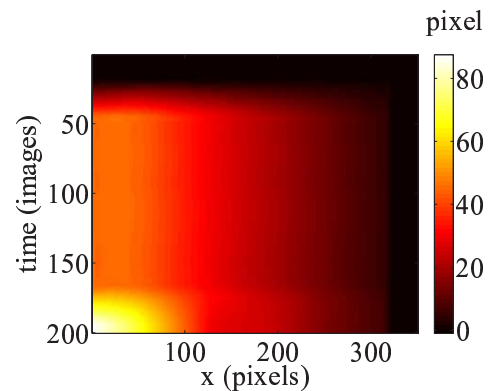

(b)

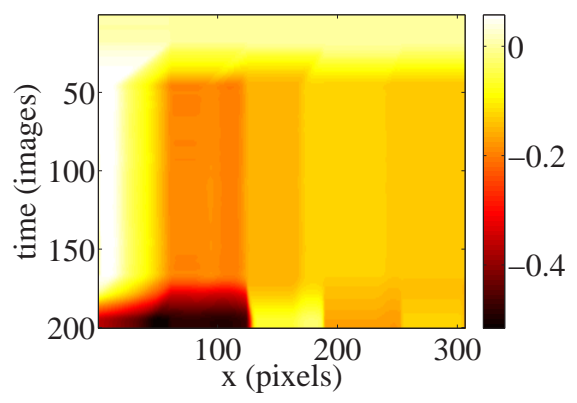

(c)

Figure 12: Velocity (a), displacement (b) and engineering strain (c) maps for the studied test. 


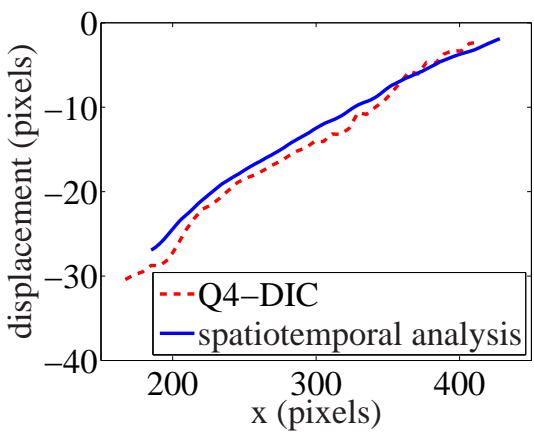

(a)

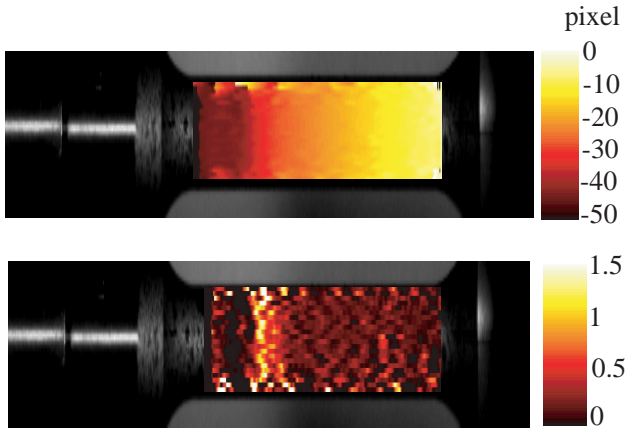

(b)

Figure 13: Comparison between the displacement field given by the spatiotemporal approach and a Q4-DIC technique (a), the element size is equal to $6 \times 6$ pixels for Q4-DIC and $4 \times 12$ pixels for the spatiotemporal approach. Displacement and engineering strain maps computed with the proposed approach for image no. 175 (just before failure) (b). 


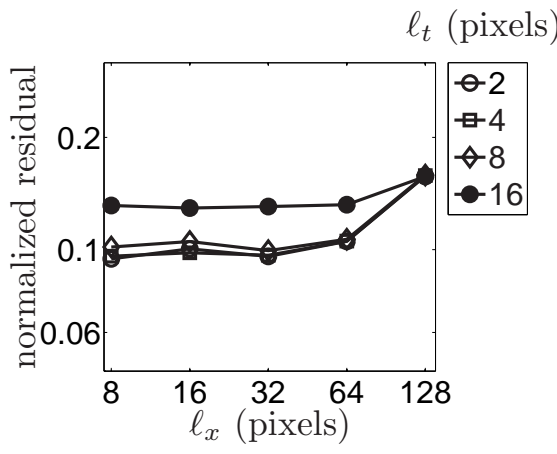

(a)

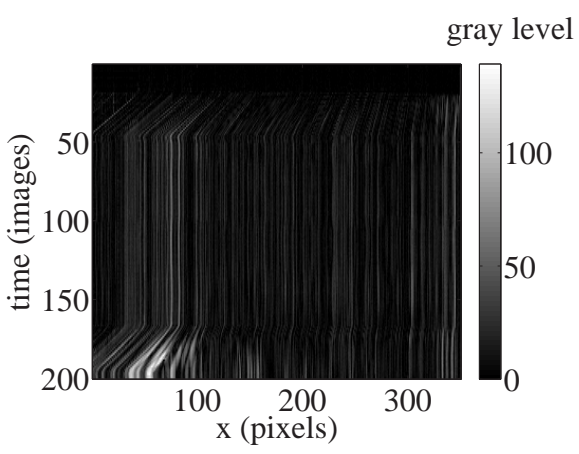

(b)

Figure 14: Normalized mean gray level residual as function of element size for the real application (a). Difference between the real and reconstructed spatiotemporal maps (b). The latter is reconstructed with the measured velocities $(4 \times 20$-pixel elements). 